\title{
Outcomes of a combined vs non-combined endoepicardial ventricular tachycardia ablation strategy
}

Daniel Matos ${ }^{1}$, Pedro Adragão ${ }^{1}$, Cristiano Pisani ${ }^{2}$, Vinicius Hatanaka ${ }^{2}$, Pedro Freitas ${ }^{1}$, Francisco Costa ${ }^{1}$, Muhieddine Chokr ${ }^{2}$, Carina $\mathrm{Hardy}^{2}$, Antonio Ferreira ${ }^{1}$, Pedro Carmo ${ }^{1}$, Sissy Lara ${ }^{2}$, Diogo Cavaco ${ }^{1}$, Francisco Morgado ${ }^{3}$, Miguel Mendes ${ }^{1}$, and Mauricio Ibrahim Scanavacca ${ }^{2}$

${ }^{1}$ Centro Hospitalar de Lisboa Ocidental EPE Hospital de Santa Cruz

${ }^{2}$ Universidade de São Paulo Instituto do Coração

${ }^{3}$ Hospital de Santa Cruz

November 26, 2020

\begin{abstract}
BACKGROUND Direct comparisons of combined (C-ABL) and non-combined (NC-ABL) endo-epicardial ventricular tachycardia (VT) ablation outcomes are scarce. We aimed to investigate the long-term clinical efficacy and safety of these 2 strategies in ischemic heart disease (IHD) and nonischemic cardiomyopathy (NICM) patients. METHODS Multicentric observational registry including 316 consecutive patients who underwent catheter ablation for drug-resistant VT between January 2008 and July 2019. Primary and secondary efficacy endpoints were defined as VT-free survival and all-cause death after ablation. Safety outcomes were defined by 30-days mortality and procedure-related complications. RESULTS Most of the patients were male (85\%), with IHD (67\%) and mean age of $63 \pm 13$ years. During a mean follow-up of $3 \pm 2$ years, 117 (37\%) patients had VT recurrence and $73(23 \%)$ died. Multivariate survival analysis identified electrical storm (ES) at presentation, IHD, left ventricular ejection fraction (LVEF), New York Heart Association (NYHA) class III/IV, and C-ABL as independent predictors of VT recurrence. In 135 patients undergoing repeated procedures, only C-ABL and ES were independent predictors of relapse. The independent predictors of mortality were C-ABL, ES, LVEF, age and NYHA class III/IV. C-ABL survival benefit was only seen in patients with a previous ablation ( $\mathrm{P}$ for interaction=0.04). Mortality at 30-days was similar between NC-ABL and $\mathrm{C}-\mathrm{ABL}(4 \%$ vs. $2 \%$, respectively, $\mathrm{P}=0.777)$, as was complication rate $(10.3 \%$ vs. $15.1 \%$ respectively, $\mathrm{P}=0.336)$. CONCLUSION A combined endo-epicardial approach was associated with greater VT-free survival and lower all-cause death in IHD and NICM patients undergoing repeated VT catheter ablations. Both strategies seem equally safe.
\end{abstract}

\section{Outcomes of a combined vs non-combined endo-epicardial ventricular tachycardia ablation strategy}

Daniel Matos $^{1^{*}}, M D$; Pedro Adragão ${ }^{1}, \mathrm{MD}$, Cristiano Pisani ${ }^{2}, \mathrm{MD}, \mathrm{PhD}$; Vinicius Hatanaka ${ }^{2}$, MD; Pedro Freitas $^{1}$, MD; Francisco Costa ${ }^{1}$, MD; Muhiedinne Chokr ${ }^{2}$, MD; Carina Hardy ${ }^{2}$, MD; António Ferreira ${ }^{1}$, MD; Pedro Carmo ${ }^{1}$, MD; Sissy Laura ${ }^{2}$, MD; Diogo Cavaco ${ }^{1}$, MD; Francisco Morgado ${ }^{1}$, MD; Miguel Mendes ${ }^{1}$, MD; Maurício Scanavacca², MD, PhD;

(1) Cardiology and Eletrophysiology Department, Hospital de Santa Cruz, Centro Hospitalar de Lisboa Ocidental, Portugal

(2) Heart Institute (Incor), University of São Paulo Medical School, São Paulo, Brazil

Corresponding author*. 
Cardiology Department, Hospital de Santa Cruz

Av. Prof. Reinaldo dos Santos 2790-134 Carnaxide, Portugal,

Tel: +351938659215

Fax: +351214188095

E-mail: danieljnmatos@gmail.com

The authors report no financial relationships or conflicts of interest regarding the content herein.

Funding: (None)

This paper has 3147 words

\section{BACKGROUND}

Direct comparisons of combined (C-ABL) and non-combined (NC-ABL) endo-epicardial ventricular tachycardia (VT) ablation outcomes are scarce. We aimed to investigate the long-term clinical efficacy and safety of these 2 strategies in ischemic heart disease (IHD) and nonischemic cardiomyopathy (NICM) patients.

\section{METHODS}

Multicentric observational registry including 316 consecutive patients who underwent catheter ablation for drug-resistant VT between January 2008 and July 2019. Primary and secondary efficacy endpoints were defined as VT-free survival and all-cause death after ablation. Safety outcomes were defined by 30-days mortality and procedure-related complications.

\section{RESULTS}

Most of the patients were male (85\%), with IHD (67\%) and mean age of $63 \pm 13$ years. During a mean followup of $3 \pm 2$ years, $117(37 \%)$ patients had VT recurrence and $73(23 \%)$ died. Multivariate survival analysis identified electrical storm (ES) at presentation, IHD, left ventricular ejection fraction (LVEF), New York Heart Association (NYHA) class III/IV, and C-ABL as independent predictors of VT recurrence. In 135 patients undergoing repeated procedures, only C-ABL and ES were independent predictors of relapse. The independent predictors of mortality were C-ABL, ES, LVEF, age and NYHA class III/IV. C-ABL survival benefit was only seen in patients with a previous ablation ( $\mathrm{P}$ for interaction=0.04). Mortality at 30 -days was similar between NC-ABL and C-ABL ( $4 \%$ vs. $2 \%$, respectively, $\mathrm{P}=0.777)$, as was complication rate $(10.3 \%$ vs. $15.1 \%$ respectively, $\mathrm{P}=0.336)$.

\section{CONCLUSION}

A combined endo-epicardial approach was associated with greater VT-free survival and lower all-cause death in IHD and NICM patients undergoing repeated VT catheter ablations. Both strategies seem equally safe.

\section{ETHICAL STANDARDS:}

All human and animal studies have been approved by the appropriate ethics committee and have therefore been performed in accordance with the ethical standards laid down in the 1964 Declaration of Helsinki and its later amendments.

The patients signed an informed consent both for the procedure and publication of any relevant data

\section{INTRODUCTION}

Patients with ischemic (IHD) and nonischemic (NICM) dilated heart disease and reduced left ventricular ejection fraction are at increased risk of ventricular tachycardias (VTs) or sudden cardiac death ${ }^{1}$. Implantable cardioverter-defibrillators (ICDs) are indicated in these patients and have shown to reduce mortality ${ }^{1}$. However, some studies suggest that ICD shocks reduce the quality of life and may be linked to increased 
mortality, particularly after an electrical storm $(\mathrm{ES})^{2}$. VT catheter ablation is an invasive treatment modality for antiarrhythmic drugs-resistant VT that reduces arrhythmic episodes, improves quality of life and improves survival in patients with $\mathrm{ES}^{3}$. Current guidelines for VT ablation ${ }^{3}$ recommend epicardial catheter ablation for NICM patients after a first failed endocardial catheter ablation or as first intention when there is a suspicion of an epicardial circuit. Epicardial ablation role is not as established in the IHD population, although some studies show potential VT-free survival improvement after a combined endo-epicardial approach $(\mathrm{C}-\mathrm{ABL})^{4}$. However, complex arrhythmia substrates and potential life-threating procedure complications increase the technical difficulty of the epicardial catheter ablation ${ }^{5}$. Direct comparisons of combined and non-combined endo-epicardial ablations outcomes are limited by patient characteristics, follow-up duration, protocols heterogeneity and scarcity of randomized trials ${ }^{4,5}$. We aim to investigate the long-term clinical outcomes of these 2 strategies in the IHD and NICM populations. To overcome said limitations, a propensity score-matched sensitivity analysis was performed.

\section{METHODS}

\section{Population}

This study included all consecutive patients with drug-resistant VT undergoing catheter ablation in two different institutions, between January 2008 and July 2019. Only patients with scar-related VT etiology of either IHD or NICM etiology were included, and no Chagas disease patient was included in this analysis. ES was defined as the occurrence of [?]3 episodes of VT or ventricular fibrillation during a 24-h period resulting in an appropriate ICD therapy.

\section{Catheter ablation protocol}

All patients underwent the procedure under arterial blood pressure and $\mathrm{O} 2$ saturation monitoring. Conscious sedation or general anesthesia were used according to the operator's discretion and with anesthesiologist support. Antiarrhythmic drugs (AAD) were stopped at least 48 hours before the procedure when applicable. The choice of the VT ablation access (endocardial vs epicardial vs combined) was decided according to the etiology, previous VT ablation site and imaging information regarding scar localization. Systemic anticoagulation with intravenous heparin targeted a minimum activation clotting time of $300 \mathrm{~s}$ during each left ventricular endocardial ablation, with protamine reversal $(1 \mathrm{mg} / 100 \mathrm{U})$ at the end of the procedure. Systemic anticoagulation was also reversed with protamine before percutaneous epicardial access when necessary. Epicardial access was obtained through the subxiphoid space under fluoroscopic guidance as previously described by Sosa et all ${ }^{6}$. A mapping multipolar catheter was placed in the coronary sinus via femoral vein and standard transvenous multipolar catheters were placed into the appropriate cardiac chambers under study. Left ventricular mapping was performed via the retrograde aortic or transseptal route. Endocardial and epicardial electroanatomical mapping was obtained with the CARTO(r) (Biosense Webster, Diamond Bar, CA, USA), the EnSite NavX(r) (St Jude Medical, St Paul, MN, USA) or with the Rhythmia(r) (Boston Scientific, Marlborough, Massachusetts, USA) systems and included activation and voltage mapping acquired in sinus rhythm, ventricular pacing or during hemodynamically stable VT. Intracardiac signals were filtered at 30 to $500 \mathrm{~Hz}$. Normal tissue was defined by a voltage threshold greater than $1.5 \mathrm{mV}$ while dense scare as tissue under a threshold of $0.5 \mathrm{mV}$ in bipolar substrate map. A first attempt to induce the clinical VT was always performed at the beginning of each procedure. Activation and entrainment-mapping techniques were performed in all hemodynamically tolerated VTs, and if not possible, ablation was guided by substrate, pace mapping and identification of delayed and fractionated potentials. Coronary angiography was performed when deemed necessary before epicardial radiofrequency applications. Epicardial phrenic nerve capture was identified by bipolar pacing from the ablation catheter at $20 \mathrm{~mA}$ with a pulse width of $10 \mathrm{~ms}$. Radiofrequency $(\mathrm{RF})$ was delivered with a 3.5-mm open irrigated tip catheter in power control mode through the use of a Stockert generator, with power set to $30-50 \mathrm{~W}$ and irrigation set to $17-30 \mathrm{ml} / \mathrm{min}$. The procedure was deemed as successful if no VT was induced with a standard stimulation protocol of up to 3 extrastimuli at a 200ms cycle length in 2 different sites, excluding induction of VF or fast polymorphic VT.

\section{Study endpoints}


The primary efficacy endpoint was defined as VT recurrence, which included any ICD appropriate therapy (antitachycardia pacing or shock) or a documented sustained VT not detected by the ICD. The secondary efficacy endpoint was defined as all-cause death. The safety outcomes were defined as 30-day mortality and procedure-related complications.

\section{Follow-up}

The follow-up protocol comprised outpatient visits on the 1st, 3rd, 6th, and 12th months' post-ablation, followed by regular assessments according to the assistant physician's discretion. Patient's data and outcomes were collected from electronic and physical medical records. If said records lacked all the required information, a structured telephonic interview was conducted. An ICD was implanted in all patients before discharge if not previously done. After the ablation, all ICDs were programmed with at least one zone of VT detection slower than the clinical VT.

\section{Statistical analysis}

Normally and non-normally distributed variables were expressed as mean and median, respectively. Differences between groups were assessed using independent samples t-test and Chi-square test for continuous and categorical variables, respectively. Proportional-hazards Cox regression was used to identify predictors of time to VT or death. Variables with a P-value (P) [?] 0.10 in univariate analysis were entered simultaneously in the multivariate regression model and deemed as statistically significant if $\mathrm{P}<0.05$. Multicollinearity was excluded by assessing Pearson's correlation coefficient between pairs of continuous variables. Kaplan-Meier curves were used to report VT-free survival for the NC-ABL and C-ABL groups, while differences in their survival curves were assessed with the log-rank test. Annual relapse rates were obtained by dividing the total numbers of first events by the total number of person-years of follow-up for each group. The propensity score (PS) for an individual is the probability of receiving a particular treatment based on a particular set of individual covariates ${ }^{7}$. A PS matching was assessed for the ablation strategy (C-ABL vs NC-ABL) by multivariable logistic regression, with the inclusion of the covariates identified as independent predictors of VT recurrence and mortality: age; IHD etiology; left ventricular ejection fraction (LVEF); New York Heart Association (NYHA) functional class III or IV; ES at presentation. The resulting scores were matched in a 1:1 ratio to the best corresponding patient, with a maximal allowable difference of 0.05 (caliper width of 0.05 of the standard deviation of the logit of the PS). Any remaining differences between matched pairs were assessed by standardized difference of the means (level of significance $<0.05$ ). Statistical analysis was performed using Statistical Package for Social Sciences (SPSS) version 23.0 (SPSS Inc., Chicago, USA) for Windows OS. Statistical significance was set at $\mathrm{P}<0.05$ (two-sided tailed).

\section{RESULTS}

\section{Population}

Baseline characteristics are presented in table 1. In a population of 316 patients, most were of male sex $(\mathrm{N}=267,85 \%)$, had ischemic cardiomyopathy $(\mathrm{N}=195,67 \%)$ and a mean age of $63+-13$ years. Mean LVEF was $34+-11 \%$. One hundred twenty-seven (40\%) patients were in either NYHA class III or IV and $84(27 \%)$ patients had an ES at presentation. During a mean follow-up of $3+-2$ years, 117 (37\%) patients had VT recurrence and $73(23 \%)$ patients died. All except one death was of cardiovascular origin (one acute biliary pancreatitis was responsible for the only non-cardiovascular death).

\section{Catheter Ablation}

The clinical VT was of right bundle branch block-like (RBBB) morphology in 254 patients (80\%) and mean procedure duration was $4+-2$ hours. Ninety-four (30\%) patients underwent LV ablation through a transseptal approach and $179(57 \%)$ through the retro aortic route. Epicardial ablation was performed in $61(19 \%)$ patients and $53(17 \%)$ patients were subject to a combined endocardial and epicardial ablation (C-ABL), either simultaneously $(\mathrm{N}=12)$ or at different procedures $(\mathrm{N}=41)$ (figure 1). One hundred and five (33\%) patients underwent more than one ablation. Only 12 patients were treated with a C-ABL in the first procedure, with the remaining having mostly 1 previous (redo) ablation. The mean number of ablations was 
$1+-1$, from 1 to 4 procedures, and most of the patients were only treated with one ablation $(\mathrm{N}=211,67 \%)$. Only six $(2 \%)$ patients underwent 3 or 4 procedures. At the end of the procedure, complete success was achieved in $83 \%$ of the patients, while only partial success in $14 \%$ of the patients and in $3 \%$ the ablations were unsuccessful in eliminating the clinical VT. The mean duration of hospital stay was of $12+-11$ days.

\section{Primary efficacy endpoint}

Regarding the arrhythmic recurrence, multivariate Cox survival analysis identified ES at presentation, NYHA class III or IV, LVEF, IHD and C-ABL as in independent predictors of relapse (table 2). The VT-free survival improvement was only statistically significant in patients with a previous ablation procedure (P for interaction $=0.003)$ in sub-group analysis. This finding was consistent in both IHD and NICM patients $(\mathrm{P}$ for interaction $=0.170)$. In patients undergoing two or more procedures $\mathrm{C}-\mathrm{ABL}(\mathrm{HR}=0.36,95 \% \mathrm{CI} 0.17-0.80$, $\mathrm{P}=0.011)$ and $\mathrm{ES}(\mathrm{HR}=2.42,95 \% \mathrm{CI} 1.24-4.70, \mathrm{P}=0.009)$ were the only independent predictors of arrhythmia recurrence.

\section{Secondary efficacy endpoint}

The independent predictors of mortality identified by survival regression analysis were ES at presentation, NYHA class III or IV, age, LVEF, IHD and C-ABL (Table 3).

In sub-group analysis, the survival improvement with a combined strategy was only statistically significant in patients undergoing a redo procedure $(\mathrm{P}$ for interaction $=0.04)$. As in the primary efficacy endpoint, this finding was consistent in both IHD and NICM patients $(\mathrm{P}$ for interaction $=0.231$ ).

\section{Safety Outcomes}

Mortality at 30-days after index-procedure was similar between NC-ABL and C-ABL (10 vs. 1 for NC-ABL and $\mathrm{C}-\mathrm{ABL}$, respectively, $\mathrm{P}=0.777)$. The complication rate was not different between both groups $(10.3 \%$ vs. $15.1 \%$ respectively, $\mathrm{P}=0.336$ ). In a sub-group analysis, the $\mathrm{C}-\mathrm{ABL}$ group had more pericardial effusions ( $2 \%$ vs $9 \%, \mathrm{P}=0.014)$ and right ventricular punctures $(1 \%$ vs $7 \%, \mathrm{P}=0.017)$. There were 2 strokes in the NC-ABL group and non in the $\mathrm{C}-\mathrm{ABL}$ group $(\mathrm{P}=1.000)$. Two patients in the NC-ABL group developed complete heart block vs. 1 in the $\mathrm{C}-\mathrm{ABL}$ group $(\mathrm{P}=1.000)$.

\section{PS matching sensitivity analysis}

A PS was used to match patients in a 1:1 fashion for NC-ABL vs C-ABL groups accordingly to all variables identified as independent predictors of VT recurrence and mortality. The PS utilized the covariates: ES at presentation, LVEF, NYHA class III/IV, IHD and age. The PS matched two groups of 43 patients. NC-ABL and C-ABL patients had a mean age of $60+-14$ and. 61 +- 12 years, being $86 \%$ and $77 \%$ of male sex and $72 \%$ being redo procedures in both groups, respectively. Both groups presented with well-matched baseline characteristics (Table 4), except for atrial fibrillation, with a higher incidence in the NC-ABL group $(\mathrm{N}=10,23 \%$ vs $\mathrm{N}=2,5 \%, \mathrm{P}=0.026)$. Only 2 patients underwent epicardial ablation at index procedure in the NC-ABL group, while $37(86 \%)$ patients were treated with an epicardial ablation and $6(14 \%)$ patients had a previous epicardial ablation in the $\mathrm{C}-\mathrm{ABL}$ group $(\mathrm{P}<0.001)$. During a mean follow-up of $3+-2$ years, $27(63 \%)$ NC-ABL patients had VT recurrence and $10(23 \%)$ in the C-ABL group $(\mathrm{P}=0.003)$. The yearly rates of $\mathrm{VT}$ recurrence were $34 \% /$ year for $\mathrm{NC}-\mathrm{ABL}$ vs. $11 \% /$ year for $\mathrm{C}-\mathrm{ABL}(\mathrm{P}=0.003)$. Multivariate survival analysis in the PS population identified only $\mathrm{C}-\mathrm{ABL}(\mathrm{HR}=0.42,95 \% \mathrm{CI} 0.20-0.88, \mathrm{P}=0.023)$ as an independent predictor of VT recurrence (Figure 2) in both ischemic and nonischemic dilated cardiomyopathy $(\mathrm{P}$ for interaction $=0.110)$, but only in patients with a previous endocardial ablation $(\mathrm{P}$ for interaction $=$ $0.03)$.

\section{Safety Outcomes in the PS population}

Mortality at 30-days post-procedure was similar between NC-ABL and C-ABL (2 vs.1 for NC-ABL and $\mathrm{C}-\mathrm{ABL}$, respectively, $\mathrm{P}=0.501)$. The complication rate was not different between both groups (9.3\% vs. $14.0 \%$ respectively, $\mathrm{P}=0.738$ ) (table 4 ). There was 1 pericardial effusion in the NC-ABL group vs 4 in the $\mathrm{C}-\mathrm{ABL}$ group $(\mathrm{P}=0.167)$ and in only the $\mathrm{C}-\mathrm{ABL}$ group occurred right ventricle puncture (2 patients). Two 
patients in the NC-ABL group developed complete heart block vs. 1 in the C-ABL group $(\mathrm{P}=0.567)$ (Table $4)$.

\section{DISCUSSION}

This study documents the long-term outcomes of a C-ABL strategy in a population of IHD and NICM patients. In our multicentric real-world analysis, a C-ABL in patients undergoing a redo ablation was associated with increased overall survival and VT-free survival. Direct comparison of clinical outcomes between a NC-ABL and a C-ABL strategy for VT ablation has been limited by heterogeneous patient characteristics and lack of abundant comparative randomized trials ${ }^{4,5}$. ES at presentation, LVEF, NYHA class III/IV, IHD and C-ABL have been identified as independent predictors of VT-free survival, cardiovascular and allcause mortality in our study. IHD patients with previous myocardial infarction and low ejection fraction are at an increased risk of VT and sudden cardiac death ${ }^{1,3}$, and international guidelines recommend ICD implantation ${ }^{1}$. However, recurrent VT and ES are associated with increased mortality even in patients with an $\mathrm{ICD}^{2}$. This may be related to a progressive deterioration of cardiac function resulting from frequent shocks, chronic low-cardiac output, and AAD therapy toxicity ${ }^{8}$. Several studies have shown catheter ablation superiority compared to AADs for VT treatment, with a success rate of $51 \%$ to $67 \%^{9}$. The VANISH randomized controlled trial ${ }^{10}$ showed that in patients with IHD and an ICD with VT despite AAD therapy, VT ablation had a lower rate of the composite primary outcome of death, ventricular tachycardia storm, or appropriate ICD compared to AAD therapy escalation. An endocardial-only ablation frequently does not eliminate all the re-entrant circuits in IHD, which may lead to relapse ${ }^{11}$. Also, the development and widespread use of reperfusion therapies in the last decades led to a higher number of patients with nontransmural necrosis and heterogeneous infarcted tissues. These scars may have multiple slow conduction channels with epicardial exit sites ${ }^{12}$, providing the rationale for a C-ABL approach. Current guidelines have an IIB class recommendation for the epicardial ablation in IHD after a failed endocardial ablation ${ }^{3}$. However, there is an important knowledge gap. Regarding this cardiomyopathy, Di Biase et all ${ }^{4}$ showed that extensive scar homogenization with a combined endocardial and epicardial approach in a first ablation procedure was linked to increased VT-freedom in patients with ES. One possible explanation for their findings is a greater amount and complexity of the arrhythmic substrate in IHD with ES presentation, which can justify an initial combined approach. Izquierdo et $\mathrm{al}^{13}$ showed that a first combined endo-epicardial procedural was linked to fewer hospitalizations but failed to show an increase in survival free of VT. Sarkozy et all ${ }^{14}$ showed that two-thirds of the patients selected for epicardial mapping after a failed endocardial ablation had epicardial arrhythmic substrate and Acosta et all ${ }^{15}$ showed that an endocardial ablation in patients with transmural MI was associated with an increased risk of recurrence. Tung et all ${ }^{16}$ showed better VT-free survival for IHD patients after a combined endo-epi ablation vs. endocardial only, partly explained by the high number of previous endocardial ablation (80\%) in their series. In our study, the arrhythmic relapse reduction was only verified in the population that underwent more than one procedure. Our analysis showed an increase in VT-free survival and a decrease in all-cause death with a C-ABL in IHD patients with multiple ablations, and to the best of our knowledge, this is the first individual study to show this survival benefit. A recent meta-analysis ${ }^{17}$ was also consistent with our findings, showing a reduction in all-cause mortality and VTrecurrence in IHD. These combined findings suggest that an epicardial ablation is probably the most effective strategy for VT elimination and survival improvement after an initial failed endocardial ablation in IHD. VT arrhythmogenic substrate differs from NICM and IHD patients. An analysis of 445 patients undergoing VT ablation showed that some NICM VT's critical isthmi can't be identified in either endo and epicardium, possibly due to mid myocardial location ${ }^{18}$. Current guidelines suggest an initial endocardial ablation, or possibly a first epicardial ablation if the arrhythmia has characteristics pointing to an epicardial origin ${ }^{18}$, and NICM patients have shown VT-free survival from $41 \%$ to $70 \%$ at 1-year after the procedure ${ }^{19}$. The worse outcomes of VT ablation in NICM patients appear to be related to an intramural or epicardial localization of the myocardial isthmus ${ }^{20}$. The acute success and VT-freedom after catheter ablation in NICM patients are associated with a reduction in mortality and heart transplantation ${ }^{19}$. To the best of our knowledge, this is the first study to show an improvement in VT-free survival and reduction of all-cause death with a C-ABL compared to a NC-ABL in NICM patients undergoing a redo procedure. The fundamental PS 
matching principle is the homogenization of a chosen set of covariates according to a dependent variable, in a pseudo-randomized controlled design ${ }^{7}$. This statistic technique is of particular interest in this setting since we are presented with 2 heterogeneous groups with several potential confounding variables. PS matching allows the reduction of the impact of other independent variables in the outcome analysis, strengthing the findings of the initial analysis and allowing a more accurate assessment of the safety outcomes. Serious and potentially life-threating complications can be associated with the epicardial ablation technique ${ }^{11,14,17}$. Common procedure-related complications are right ventricular puncture, subxiphoid bleeding, pericardial effusion, cardiac tamponade, coronary arteries lesion, acute myocardial infarction, thoracic artery lesion, complete heart block, phrenic nerve lesion, abdominal organ puncture, and stroke. Our population has a high burden of traditional cardiovascular risk factors (Table 1), and there was a considerable amount of ES at presentation. Although this was a high-risk population, our complication rate was comparable to the current reports in the literature. In this real-world population analysis, the C-ABL strategy had a similar safety profile when compared to the NC-ABL approach, despite that the combined strategy featured a much higher number of epicardial ablations. While we can not completely exclude the influence of underpowering in the procedural complication rate analysis, the potential benefit of this combined ablation appears to outweigh the potential risks.

\section{Limitations}

Being a real-world retrospective observational analysis, our study lacks the randomization of potential future trials. Second, our research analyzed the populations of two high-volume nationwide referrals for VT ablation, whose results may not be generalized to all centers due to referral bias. Third, while PS matching is a viable tool for covariate homogenization, we can't guarantee the non-inclusion of other potential confounding variables. Nonetheless, the verification of similar results in the PS population strengthens its conclusions. Fourth, it was not possible to access detailed information on the ventricular scars (voltage maps or cardiac magnetic resonance imaging) in all patients. At last, the choice of combined or non-combined strategy was done at the operator's discretion, which may create a nonobjectifiable bias.

\section{CONCLUSION}

In patients undergoing repeated VT catheter ablations, a combined endo-epicardial strategy appears to be associated with greater VT-free survival and lower all-cause death. This finding is consistent in both ischemic and nonischemic dilated cardiomyopathy, and both strategies seem equally safe.

\section{BIBLIOGRAPHY:}

1. Zipes DP, Camm AJ, Borggrefe M, Buxton AE, Chaitman B, Fromer M, Gregoratos G, Klein G, Moss AJ, Myerburg RJ, et al. ACC/AHA/ESC 2006 guidelines for management of patients with ventricular arrhythmias and the prevention of sudden cardiac death: a report of the American College of Cardiology/American Heart Association Task Force and the European Society of Cardiology Committee for Practice Guidelines (Writing Committee to Develop Guidelines for Management of Patients With Ventricular Arrhythmias and the Prevention of Sudden Cardiac Death). J Am Coll Cardiol. 2006 Sep 5;48(5):e247-346.

2. Hohnloser SH, Al-Khalidi HR, Pratt CM, Brum JM, Tatla DS, Tchou P, Dorian P. Electrical storm in patients with an implantable defibrillator: incidence, features, and preventive therapy: insights from a randomized trial. Eur Heart J. 2006 Dec;27(24):3027-32.

3. Cronin EM, Bogun FM, Maury P, Peichl P, Chen M, Namboodiri N, Aguinaga L, Leite LR, Al-Khatib SM, Anter E, et al. 2019 HRS/EHRA/APHRS/LAHRS expert consensus statement on catheter ablation of ventricular arrhythmias. J Arrhythmia. 2019 Jun;35(3):323-484.

4. Di Biase L, Santangeli P, Burkhardt DJ, Bai R, Mohanty P, Carbucicchio C, Dello Russo A, Casella M, Mohanty S, Pump A, et al. Endo-epicardial homogenization of the scar versus limited substrate ablation for the treatment of electrical storms in patients with ischemic cardiomyopathy. J Am Coll Cardiol. 2012 Jul 10;60(2):132-41. 
5. Berruezo A, Acosta J, Fernandez-Armenta J, Pedrote A, Barrera A, Arana-Rueda E, Bodegas AI, Anguera I, Tercedor L, Penela D, et al. Safety, long-term outcomes and predictors of recurrence after first-line combined endoepicardial ventricular tachycardia substrate ablation in arrhythmogenic cardiomyopathy. Impact of arrhythmic substrate distribution pattern. A prospective multicentre study. Eur Eur Pacing Arrhythm Card Electrophysiol J Work Groups Card Pacing Arrhythm Card Cell Electrophysiol Eur Soc Cardiol. 2017 Apr 1;19(4):607-16.

6. Sosa E, Scanavacca M, d'Avila A, Pilleggi F. A new technique to perform epicardial mapping in the electrophysiology laboratory. J Cardiovasc Electrophysiol. 1996 Jun;7(6):531-6.

7. Rosenbaum PR, Rubin DB. The central role of the propensity score in observational studies for causal effects. Biometrika. 1983 Apr 1;70(1):41-55.

8. Poole JE, Johnson GW, Hellkamp AS, Anderson J, Callans DJ, Raitt MH, Reddy RK, Marchlinski FE, Yee R, Guarnieri T, et al. Prognostic importance of defibrillator shocks in patients with heart failure. N Engl J Med. 2008 Sep 4;359(10):1009-17.

9. Stevenson WG, Wilber DJ, Natale A, Jackman WM, Marchlinski FE, Talbert T, Gonzalez MD, Worley SJ, Daoud EG, Hwang C, et al. Irrigated radiofrequency catheter ablation guided by electroanatomic mapping for recurrent ventricular tachycardia after myocardial infarction: the multicenter thermocool ventricular tachycardia ablation trial. Circulation. 2008 Dec 16;118(25):2773-82.

10. Sapp JL, Wells GA, Parkash R, Stevenson WG, Blier L, Sarrazin JF, Thibault B, Rivard L, Gula L, Leong-Sit P, et al. Ventricular Tachycardia Ablation versus Escalation of Antiarrhythmic Drugs. N Engl J Med. 2016 Jul 14;375(2):111-21.

11. Schmidt B, Chun KRJ, Baensch D, Antz M, Koektuerk B, Tilz RR, Metzner A, Ouyang F, Kuck KH. Catheter ablation for ventricular tachycardia after failed endocardial ablation: epicardial substrate or inappropriate endocardial ablation? Heart Rhythm. 2010 Dec;7(12):1746-52.

12. Schreieck J, Zrenner B, Deisenhofer I, Schmitt C. Rescue ablation of electrical storm in patients with ischemic cardiomyopathy: a potential-guided ablation approach by modifying substrate of intractable, unmappable ventricular tachycardias. Heart Rhythm. 2005 Jan;2(1):10-4.

13. Izquierdo M, Sanchez-Gomez JM, Ferrero de Loma-Osorio A, Martinez A, Bellver A, Pelaez A, Nunez J, Nunez C, Chorro J, Ruiz-Granell R. Endo-epicardial versus only-endocardial ablation as a first line strategy for the treatment of ventricular tachycardia in patients with ischemic heart disease. Circ Arrhythm Electrophysiol. 2015 Aug;8(4):882-9.

14. Sarkozy A, Tokuda M, Tedrow UB, Sieria J, Michaud GF, Couper GS, John R, Stevenson WG. Epicardial ablation of ventricular tachycardia in ischemic heart disease. Circ Arrhythm Electrophysiol. 2013 Dec;6(6):1115-22.

15. Acosta J, Fernandez-Armenta J, Penela D, Andreu D, Borras R, Vassanelli F, et al. Infarct transmurality as a criterion for first-line endo-epicardial substrate-guided ventricular tachycardia ablation in ischemic cardiomyopathy. Heart Rhythm. 2016 Jan;13(1):85-95.

16. Tung R, Michowitz Y, Yu R, Mathuria N, Vaseghi M, Buch E, Korshunov V, Perea RJ, de Caralt TM, Ortiz JT, et al. Epicardial ablation of ventricular tachycardia: an institutional experience of safety and efficacy. Heart Rhythm. 2013 Apr;10(4):490-8.

17. Romero J, Cerrud-Rodriguez RC, Di Biase L, Diaz JC, Alviz I, Grupposo V, Cerna L, Avendano R, Tedrow U, Natale A, et al. Combined Endocardial-Epicardial Versus Endocardial Catheter Ablation Alone for Ventricular Tachycardia in Structural Heart Disease: A Systematic Review and Meta-Analysis. JACC Clin Electrophysiol. 2019 Jan;5(1):13-24.

18. Shirai Y, Liang JJ, Santangeli P, Arkles JS, Schaller RD, Supple GE, Nazarian S1, Garcia FC1, Lin D1, Dixit S, et al. Comparison of the Ventricular Tachycardia Circuit Between Patients With Ischemic and 
Nonischemic Cardiomyopathies. Circ Arrhythm Electrophysiol. 2019 Jul;12(7):e007249.

19. Tung R, Vaseghi M, Frankel DS, Vergara P, Di Biase L, Nagashima K, Yu R, Vangala S, Tseng CH, Choi EK, et al. Freedom from recurrent ventricular tachycardia after catheter ablation is associated with improved survival in patients with structural heart disease: An International VT Ablation Center Collaborative Group study. Heart Rhythm. 2015 Sep;12(9):1997-2007.

20. Nakahara S, Tung R, Ramirez RJ, Michowitz Y, Vaseghi M, Buch E, Gima J, Wiener I, Mahajan A, Boyle NG, et al. Characterization of the arrhythmogenic substrate in ischemic and nonischemic cardiomyopathy implications for catheter ablation of hemodynamically unstable ventricular tachycardia. J Am Coll Cardiol. 2010 May 25;55(21):2355-65.

Table 1: Baseline characteristics of the population

\begin{tabular}{|c|c|}
\hline Baseline characteristics & Population $(\mathrm{N}=316)$ \\
\hline Male sex - no. (\%) & $267(84.5)$ \\
\hline Age - mean $\pm S D$ & $63 \pm 13$ \\
\hline HTN - no. (\%) & $241(76.3)$ \\
\hline Dyslipidemia - no. (\%) & $234(74.1)$ \\
\hline Diabetes mellitus type $2-$ no. $(\%)$ & $78(24.7)$ \\
\hline History of tobacco consumption - no. (\%) & $161(50.9)$ \\
\hline Previous myocardial infarction - no. (\%) & $195(61.7)$ \\
\hline Previous $\mathrm{CABG}-$ no. $(\%)$ & $112(35.4)$ \\
\hline Chronic kidney disease - no. (\%) & $124(39.2)$ \\
\hline Beta-blocker - no. (\%) & $308(97.5)$ \\
\hline Amiodarone - no. (\%) & $265(83.9)$ \\
\hline ICD - no. (\%) & $294(93.0)$ \\
\hline $\operatorname{LVEF}(\%)-$ mean \pm SD & $34 \pm 11$ \\
\hline Ischemic etiology - no. (\%) & $195(61.7)$ \\
\hline RBBB-like VT morphology - no. (\%) & $254(80.4)$ \\
\hline Electrical storm at presentation - no. (\%) & $84(26.6)$ \\
\hline Hospitalization duration (days) - mean $\pm \mathrm{SD}$ & $12 \pm 16$ \\
\hline Previous ablation - no. (\%) & $105(33.2)$ \\
\hline Complications - no. (\%) & $35(11.1)$ \\
\hline Pericardial effusion - no. (\%) & $10(3.2)$ \\
\hline Right ventricle puncture - no. (\%) & $7(2.2)$ \\
\hline Vascular complication - no. (\%) & $16(5.1)$ \\
\hline Complete heart block - no. (\%) & $7(2.2)$ \\
\hline Stroke/TIA - no. (\%) & $2(0.6)$ \\
\hline Pericarditis - no. $(\%)$ & $2(0.6)$ \\
\hline
\end{tabular}




\begin{tabular}{lccc} 
Predictors of VT relapse & HR & $95 \% \mathrm{Cl}$ & P-value \\
\hline ES at presentation & 2.17 & $1.44-3.25$ & $<0.001$ \\
NYHA class III or IV & 1.79 & $1.13-2.85$ & 0.013 \\
LVEF & 0.97 & $0.95-0.99$ & 0.015 \\
IHD & 0.53 & $0.36-0.78$ & 0.001 \\
C-ABL & 0.49 & $0.27-0.92$ & 0.025 \\
\hline
\end{tabular}




\begin{tabular}{lccc} 
Predictors of all-cause death & HR & 95\% Cl & P-value \\
\hline ES at presentation & 2.17 & $1.33-3.54$ & 0.002 \\
NYHA class III or IV & 2.04 & $1.12-3.73$ & 0.021 \\
Age & 1.03 & $1.01-1.05$ & 0.013 \\
LVEF & 0.95 & $0.92-0.98$ & 0.003 \\
C-ABL & 0.22 & $0.05-0.91$ & 0.037 \\
\hline
\end{tabular}


Table 4: Baseline characteristics after propensity score matching

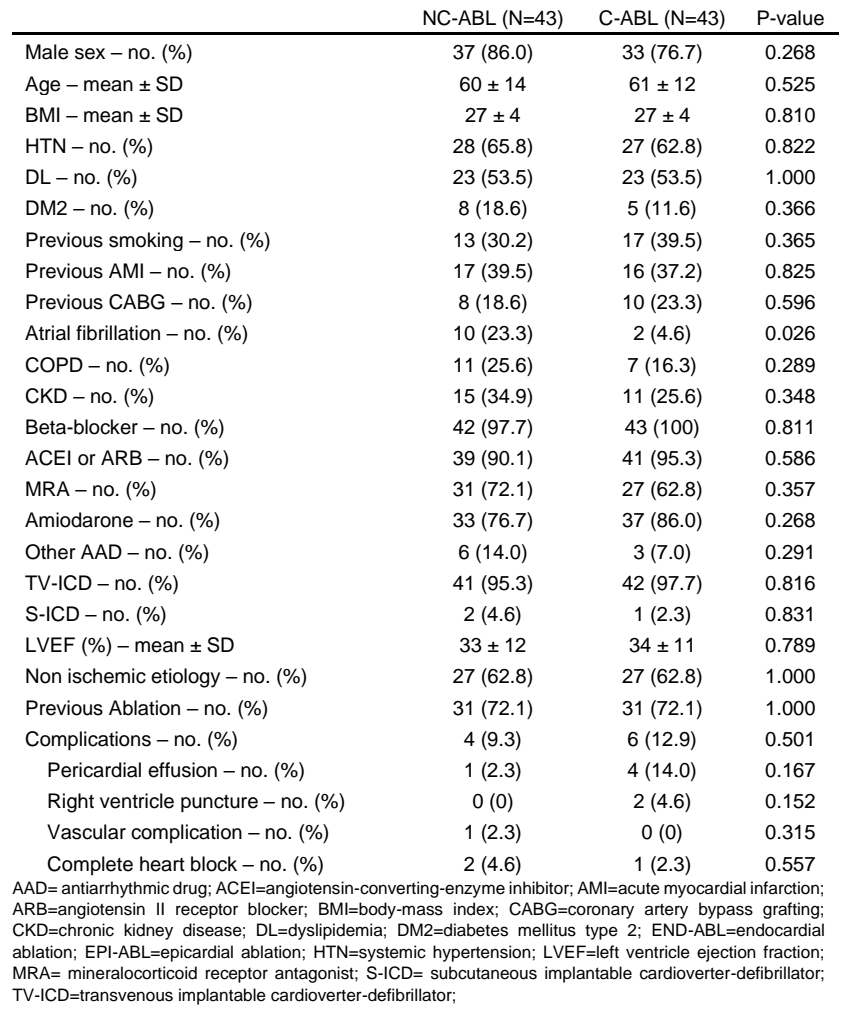



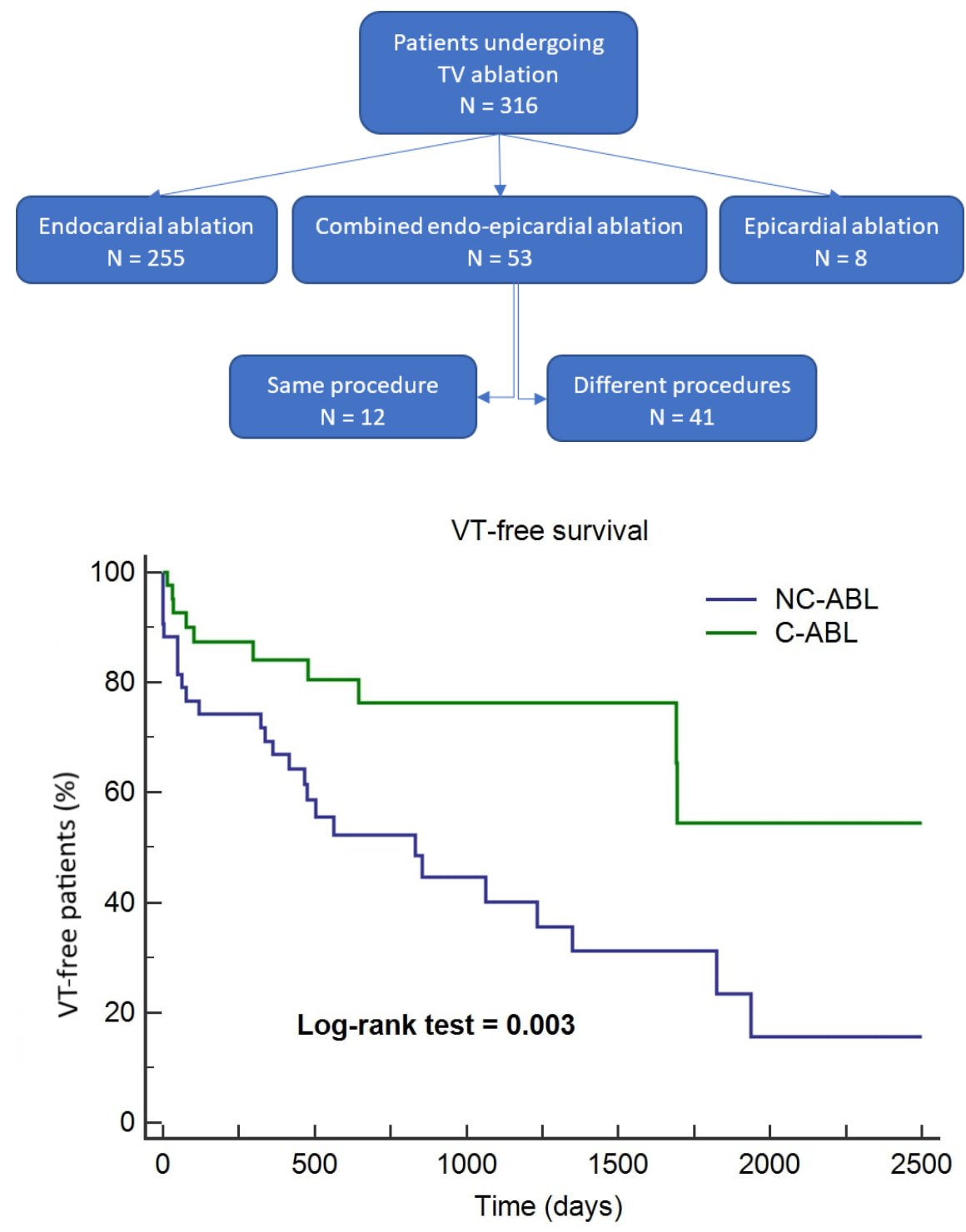